\title{
miR-155 Promotes ox-LDL-Induced Autophagy in Human Umbilical Vein Endothelial Cells
}

\author{
Zhaozhi Zhang, ${ }^{1}$ Xudong Pan, ${ }^{1}$ Shaonan Yang, ${ }^{1}$ Aijun Ma, ${ }^{1}$ Kun Wang, ${ }^{1}$ Yuan Wang, \\ Ting $\mathrm{Li}^{1}{ }^{1}$ and Shihai $\mathrm{Liu}^{2}$ \\ ${ }^{1}$ Department of Neurology, The Affiliated Hospital of the Qingdao University, Qingdao, Shandong 266100, China \\ ${ }^{2}$ Central Laboratory, The Affiliated Hospital of the Qingdao University, Qingdao, Shandong 266100, China \\ Correspondence should be addressed to Aijun Ma; drmaj@126.com
}

Received 4 November 2016; Revised 11 March 2017; Accepted 21 March 2017; Published 4 June 2017

Academic Editor: Yona Keisari

Copyright ( 2017 Zhaozhi Zhang et al. This is an open access article distributed under the Creative Commons Attribution License, which permits unrestricted use, distribution, and reproduction in any medium, provided the original work is properly cited.

\begin{abstract}
As an evolutionarily conserved metabolic process, autophagy is involved in the process of atherosclerosis (AS). MicroRNA-155 (miR-155), a multifunctional miRNA, plays an important role in many physiological and pathological conditions, including AS and autophagy. However, the effect of miR-155 on the regulation of autophagy in endothelial cells has not been reported to date. Therefore, the objective of our study was to investigate the role of miR-155 in autophagy induced by oxidized low-density lipoprotein (ox-LDL) in human umbilical vein endothelial cells (HUVECs). Our results demonstrated that ox-LDL induced autophagy in HUVECs and increased the expression of miR-155 significantly. Overexpression of miR-155 improved autophagic activity, whereas low expression of miR-155 inhibited autophagic activity. Therefore, the data demonstrated that miR-155 has a modulating effect on the autophagy of vascular endothelial cells.
\end{abstract}

\section{Introduction}

Autophagy is an evolutionarily conserved metabolic process in which denatured proteins and damaged organelles are degraded through the lysosomal system for the maintenance of intracellular homeostasis during various stress conditions $[1,2]$. The process is essential for cell growth, differentiation, and development [3]. Accumulating data have indicated that autophagy plays a potential role as diagnostic and prognostic indicators in various diseases containing AS [4-6], which is currently considered a chronic inflammatory disease of the arterial wall.

MicroRNAs (miRNAs) are a class of endogenous noncoding small RNA molecules that regulate gene expression and mediate posttranscriptional gene silencing by combining with the $3^{\prime}$-untranslaed region (3'-UTR) of the target mRNAs [7]. These RNAs are involved in the regulation of multiple cellular processes, including proliferation, differentiation, development, and apoptosis, [8] and have also been associated with AS [9-11] and autophagy $[12,13]$.
miR-155, a multifunctional miRNA, plays an important role in many physiological and pathological conditions, including AS [14, 15]. Recently, some studies have demonstrated that miR-155 could regulate the autophagy of tumor cells [16] and macrophages [17]. However, the correlation between miR-155 and autophagic activity in endothelial cells has not been reported. Therefore, the goal of this study was to investigate the role of miR-155 in autophagy mediated by ox-LDL in HUVECs.

\section{Materials and Methods}

2.1. Cell Culture. HUVECs were purchased from Genechem Co. Ltd. (Shanghai, China). Cells were cultured in Dulbecco's modified Eagle's medium (DMEM) (Hyclone, USA) supplemented with 10\% fetal bovine serum (FBS) (Tianhang Biotechnology Co. Ltd., Zhejiang, China) and $100 \mathrm{U} / \mathrm{ml}$ penicillin- $100 \mu \mathrm{g} / \mathrm{ml}$ streptomycin (Solarbio Science \& Technology Co. Ltd., Beijing, China) at $37^{\circ} \mathrm{C}$ in a humidified $5 \% \mathrm{CO}_{2}$ incubator. 


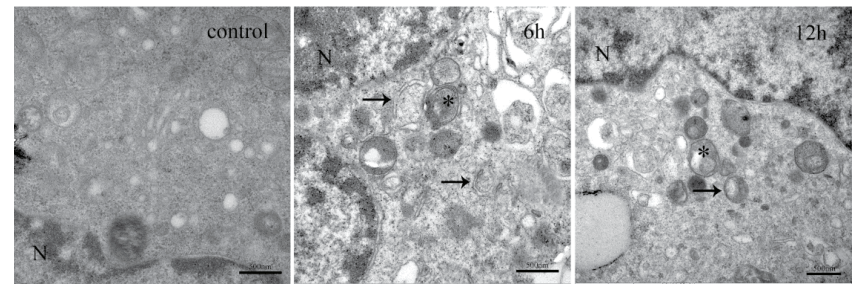

Figure 1: TEM images of HUVECs. HUVECs were exposed to ox-LDL (100 $\mu \mathrm{g} / \mathrm{ml})$ for $6 \mathrm{~h}$ and $12 \mathrm{~h}$, and cell samples were collected for TEM analysis. Autophagosomes were indicated by arrows. Autolysosomes were indicated by asterisks. N: nucleus. Scale bar $=500 \mathrm{~nm}$.

2.2. Cell Treatment. To observe the effect of ox-LDL on autophagy induction and select the optimal time point, we used $100 \mu \mathrm{g} / \mathrm{ml}$ ox-LDL (Yi Yuan Biotech, Guangzhou, China) to stimulate the HUVECs for 6 and $12 \mathrm{~h}$. Control group cells were cultured in complete medium. To demonstrate the role of miR-155 in autophagy, we divided the cells into five groups: the control, ox-LDL, ox-LDL+miR155 mimic, ox-LDL+miR-155 inhibitor, and ox-LDL +miR-155 negative control (NC). Cells were transfected with miR-155 mimics, miR-155 inhibitors, and miR-155 NC using Lipofectamine 2000 (Invitrogen, USA) according to the manufacturer's protocol. Twenty-four hours after transfection, cells were exposed to ox-LDL $(100 \mu \mathrm{g} / \mathrm{ml})$. The exposure time was the optimal time point selected by the experiment. Control group cells were cultured in complete medium.

2.3. Transmission Electron Microscope. Cells harvested by trypsinization were fixed with $3 \%$ glutaraldehyde and postfixed in $1 \%$ osmium tetroxide. After dehydration in a graded series of ethanol, cells were embedded in Araldite. Next, $50 \mathrm{~nm}$ ultrathin sections were placed on nickel grids and subsequently stained with uranyl acetate and lead citrate.

\subsection{Quantitative Reverse Transcription Polymerase Chain} Reaction ( $q R T-P C R)$. Total RNA was extracted from cells using Trizol reagent (Invitrogen) according to the manufacturer's protocol. qRT-PCR analysis for miRNAs was performed by using a miRNA cDNA Synthesis Kit and miRNA qPCR Assay Kit (ComWin Biotech Co. Ltd., Beijing, China). qRT-PCR analysis for mRNAs was conducted according to manufacturer's instructions using a PrimeScript $^{\mathrm{TM}}$ RT reagent Kit and SYBR ${ }^{\circledR}$ Premix Ex Taq ${ }^{\mathrm{TM}}$ (Takara, Japan). All primers used in the study were obtained from Sangon Biotech, Shanghai. Relative expression levels were calculated using the $2^{-\triangle \Delta \mathrm{Ct}}$ method. The mRNA level of GAPDH was used as an internal control.

2.5. Western Blot. Cells were washed twice with PBS and then lysed in RIPA buffer (Takara, Japan) containing the protease inhibitors. After centrifugation at $12000 \mathrm{~g}$ for 20 minutes at $4^{\circ} \mathrm{C}$, total protein concentration in the supernatant was quantified using the BCA protein assay kit (Comwin Biotech Co. Ltd., Beijing, China). Protein samples were separated by SDS-PAGE electrophoresis and then transferred onto PVDF membranes (Millipore, USA). The membranes were blocked with 5\% nonfat milk powder-TBS-Tween 20 for 2 hours at room temperature and then incubated with $1: 1000$-diluted primary antibodies against LC3 and p62 (Cell Signaling Technology, USA) overnight at $4^{\circ} \mathrm{C}$. Then, membranes were briefly washed thrice with TBST and incubated with $1: 10000$-diluted horseradish peroxidase-conjugated secondary antibody (Abcam, USA) for an additional one hour at room temperature. After washing thrice with TBST, the membranes were visualized using an ECL Western Blotting Substrate Kit (Millipore, USA) and the GelDoc XR Gel Documentation System (BioRad, USA). The gray value was analyzed by ImageJ software.

2.6. Statistical Analyses. Data were presented as the mean \pm SD for at least three sets of separate experiments. Differences between groups were assessed using one-way ANOVA with a Tukey correction. Statistical analysis was performed using SPSS 19.0 for Windows software (SPSS Inc., Chicago, IL). $p<0.05$ was considered to be statistically significant.

\section{Results}

3.1. Autophagy Induced by ox-LDL in HUVECs. To observe the autophagic activity, we used transmission electron microscopy (TEM) to detect the autophagosomes and autolysosomes in cells. The autophagosome, a special vesicle with a double membrane structure, is an important marker of autophagy activation, which ultimately fuse with the lysosome to form the autolysosome, leading to the degradation of cellular structures [18]. The control group image displayed few autophagosomes and autolysosomes. In contrast, cells treated with ox-LDL for 6 and $12 \mathrm{~h}$ exhibited many typical autophagosomes and autolysosomes in the cytoplasm, and the number of autophagosomes at $6 \mathrm{~h}$ was much more than that at $12 \mathrm{~h}$ (Figure 1). LC3-II is a well-known marker of autophagy. p62, a polyubiquitin-binding protein, is selectively incorporated into autophagosomes through direct binding to LC3 and efficiently degraded during autophagy [19]. To further confirm autophagy, we observed whether LC3-II and p62 were regulated in response to stimulation by ox-LDL. The results showed that ox-LDL increased the expression of LC3-II but decreased the expression of p62, as shown in Figure 2. And the level of LC3-II at $6 \mathrm{~h}$ was much higher than that at $12 \mathrm{~h}(p<0.05)$, whereas the level of $\mathrm{p} 62$ at $6 \mathrm{~h}$ was significantly lower $(p<0.05)$. Therefore, we choose $6 \mathrm{~h}$ as the optimal time point for the subsequent experiments. 


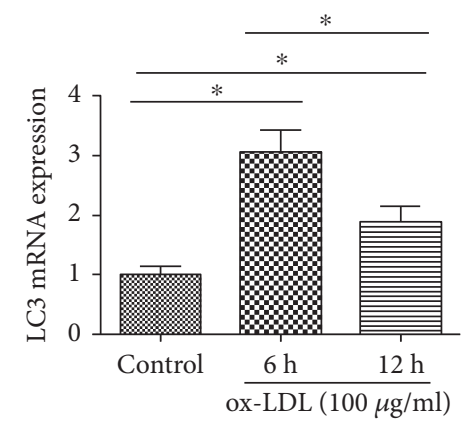

(a)

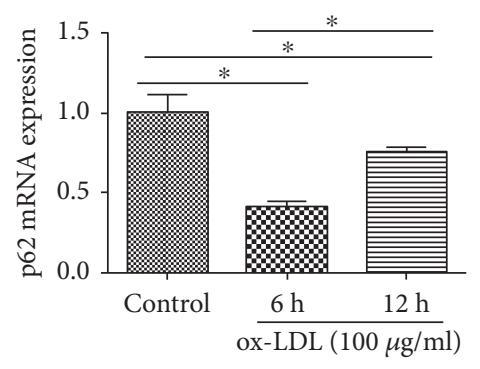

(c)

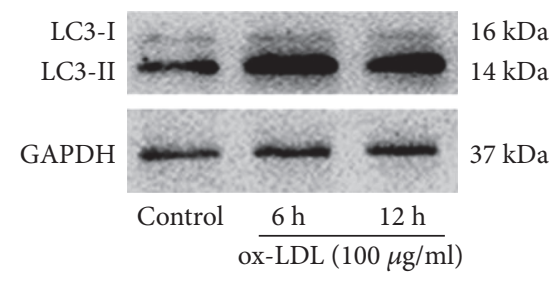

(b)
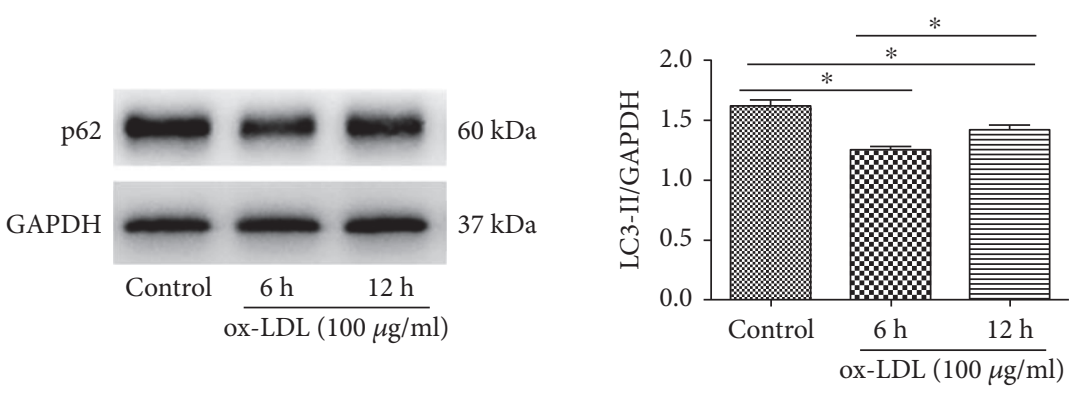

b)

(d)

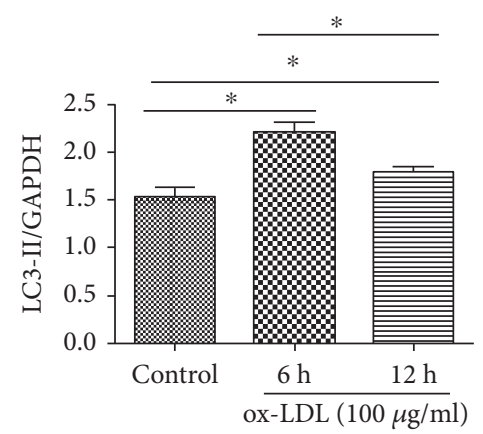

FiguRE 2: ox-LDL regulates LC3 and p62 expression. HUVECs were treated with ox-LDL ( $100 \mu \mathrm{g} / \mathrm{ml})$, and cells are collected at $6 \mathrm{~h}$ and $12 \mathrm{~h}$ for qRT-PCR and Western blot. (a, c) qRT-PCR analysis of LC3 and p62. Results are the means \pm SD of three separate experiments, ${ }^{*} p<0.05$. (b, d) Western blot analysis of LC3-II and p62. Left panels present representative blots of LC3-II and p62; right bar graphs showed statistics of optical density measurements. Results are the means \pm SD of three separate experiments, ${ }^{*} p<0.05$.

3.2. ox-LDL induced miR-155 Upregulation. To explore the relationship between ox-LDL and miR-155, we measured miR-155 expression levels in cells treated with ox-LDL $(100 \mu \mathrm{g} / \mathrm{ml})$ for 6 and $12 \mathrm{~h}$. As shown in Figure 3, compared with the control group, miR-155 expression levels in the ox-LDL group were significantly increased $(p<0.05)$. The data demonstrated that ox-LDL induced the upregulation of miR-155 in HUVECs.

\section{3. miR-155 Regulates Autophagy Induced by ox-LDL in HUVECs}

3.3.1. Effect of miR-155 on the Ultrastructure Changes of Cells. From the TEM results (Figure 4), the control group displayed no autophagosomes and autolysosomes. Compared with the control group, single treatment with ox-LDL exhibited apparent autophagosomes and autolysosomes. When cells were transfected with $\mathrm{miR}-155$ mimic, the number of autophagosomes and autolysosomes further increased, whereas transfection with the miR-155 inhibitor reduced the number of autophagosomes and autolysosomes.

\subsubsection{Effect of $m i R-155$ on LC3 and $p 62$ Expression. qRT-PCR} results showed that the upregulation of miR-155 enhanced LC3 mRNA levels, whereas the downregulation of miR155 reduced LC3 mRNA levels (Figure 5(a)). Western blot results indicated that the $\mathrm{miR}-155$ mimic improved the LC3-II/GAPDH ratio, but the miR-155 inhibitor decreased

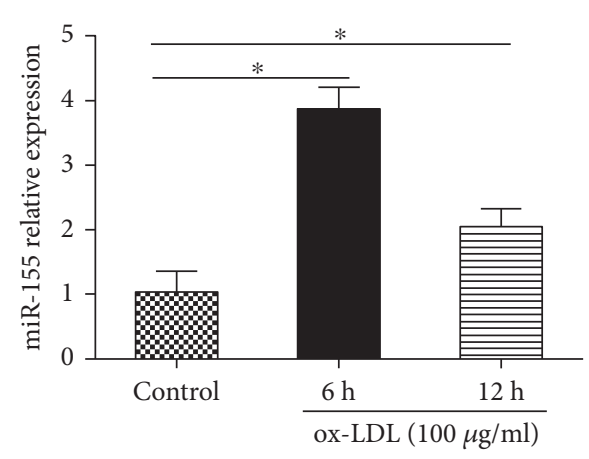

FIgURE 3: ox-LDL upregulated miR-155. HUVECs were exposed to ox-LDL $(100 \mu \mathrm{g} / \mathrm{ml})$ for 6 and $12 \mathrm{~h}$. Cells were harvested for qRT-PCR to quantify miR-155 expression. Results are the means \pm SD of three separate experiments, ${ }^{*} p<0.05$.

the ratio of LC3-II/GAPDH (Figure 5(b)). We determined p62 mRNA expression levels, and the results showed that p62 mRNA levels were reduced by the miR-155 mimic but improved by the miR-155 inhibitor (Figure 5(c)). In addition, miR-155 enhancement downregulates the ratio of p62/GAPDH, whereas miR-155 suppression upregulated the p62/GAPDH ratio (Figure 5(d)). Taken together, the data above indicated that the overexpression of miR-155 increased the autophagy level; however, the inhibition of miR-155 suppressed the level of autophagy. 

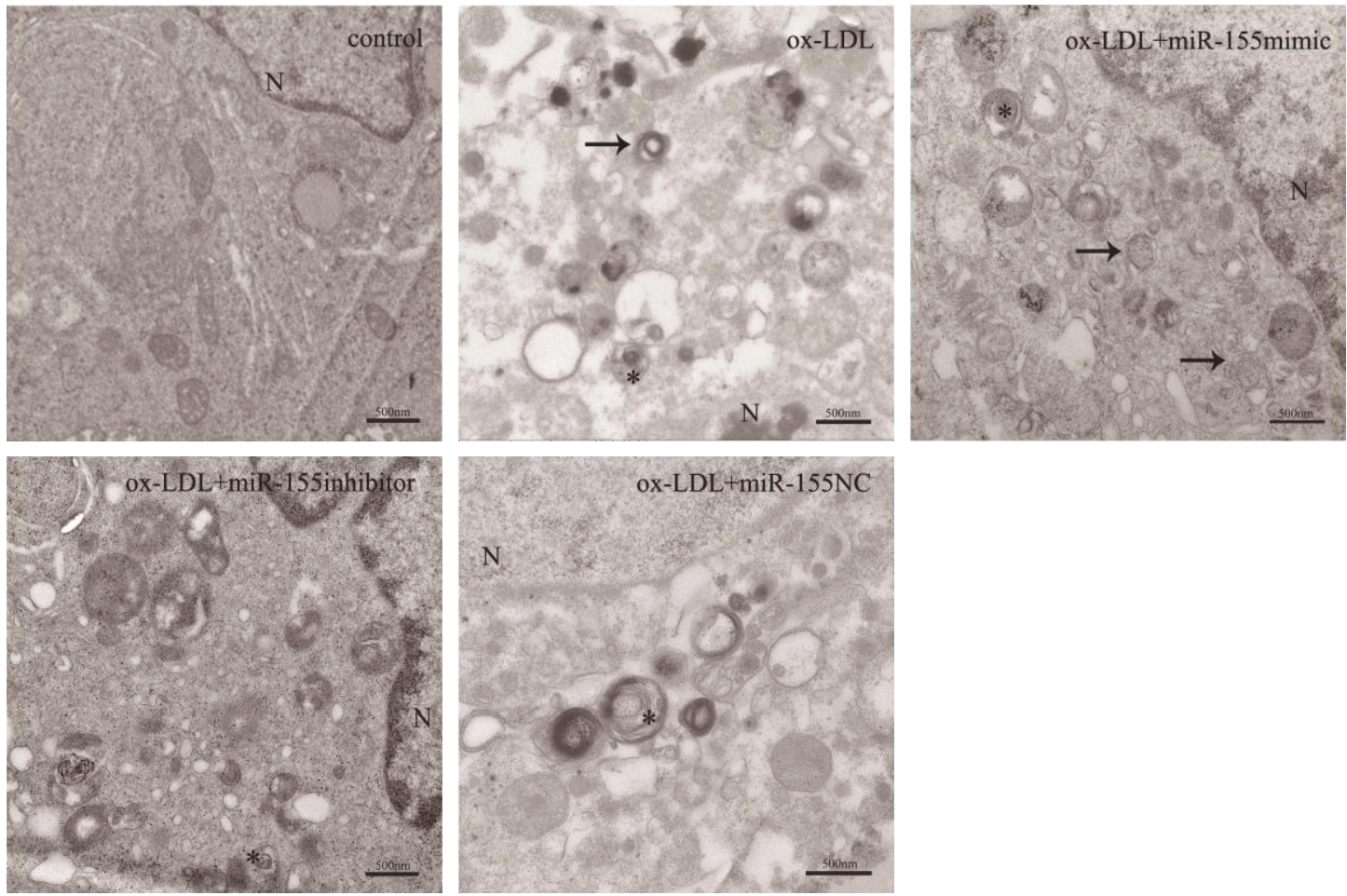

FIgURE 4: miR-155 promotes autophagosome and autolysosome accumulation. After transfection with miR-155 mimics, miR-155 inhibitors, and miR-155 NC, cells were exposed to $100 \mu \mathrm{g} / \mathrm{ml}$ ox-LDL for $6 \mathrm{~h}$ and collected for TEM analysis. Autophagosomes were indicated by arrows. Autolysosomes were indicated by asterisks. N: nucleus. Scale bar $=500 \mathrm{~nm}$.

\section{Discussion}

As a risk factor of AS, ox-LDL can promote endothelial cell injury. Previous studies have indicated that ox-LDL can result in autophagy in endothelial cells. Nowicki et al. [20] reported that many autophagosomes with double membranes were observed in the EA.hy926 endothelial cells treated with ox-LDL for $6 \mathrm{~h}$. After $12 \mathrm{~h}$ of ox-LDL stimulation, cells revealed many autophagic vacuoles with remnants of organelle inclusions. However, there were numerous large vacuoles after $24 \mathrm{~h}$, but seldom with remnants of organelle inclusions. In addition, Zhang et al. [21] found that ox-LDL upregulated the expression of LC3-II and beclin 1 in umbilical vein endothelial cells, and the upregulation of proteins reached two peaks at 0.5 and $6 \mathrm{~h}$ and then declines at $48 \mathrm{~h}$. Similarly, our study found that ox-LDL $(100 \mu \mathrm{g} / \mathrm{ml})$ increased the number of autophagosomes and the level of the protein LC3-II yet decreased the level of protein p62, which demonstrates that ox-LDL induces autophagy in HUVECs. The present study also confirmed that ox-LDL induced the upregulation of miR-155 in cells. At $6 \mathrm{~h}$ and $12 \mathrm{~h}$ after ox-LDL $(100 \mu \mathrm{g} / \mathrm{ml})$ treatment, increased miR155 expression levels were detected. This result suggested that miR-155 might have a regulatory effect on ox-LDLinduced autophagy. Therefore, to further explore the role of miR-155 in autophagy, we intervened the miR-155 level through transfecting the miR-155 mimics and inhibitors into cells. The results showed that the miR-155 mimic increased the number of autophagic bodies and the ratio of LC3-II/ GAPDH yet decreased the expression of p62. In contrast, the miR-155 inhibitor downregulated the number of autophagosomes and the expression level of LC3-II but improved p62 expression levels. As a result, the collective data demonstrated that miR-155-enhanced autophagy induced by ox-LDL.

In previous studies, the correlation between miR-155 and autophagy has been studied in other cell types. Chen et al. [16] reported that the overexpression of miR-155 induces the activation of autophagy, which promotes tumor cell survival and chemoresistance. Wang et al. [17] reported that miR-155 promotes autophagy in macrophages by targeting Rheb (a negative regulator of autophagy), conferring protection against infection with intracellular mycobacteria. In addition, $\mathrm{Wu}$ et al. [22] demonstrated that the overexpression of miR-155 by transfection with miR-155 mimics significantly decreased the survival of intracellular Helicobacter pylori in human gastric epithelial cells, and this process was mediated by the induction of autophagy. However, the relationship between miR-155 and autophagy in endothelial cells has not been reported. Our present results demonstrate that miR-155 promotes ox-LDL-induced autophagy in HUVECs.

Moreover, miR-155 plays a crucial role in AS development, which involves multiple mechanisms. Huang et al. [23] demonstrated that miR-155 serves as a negative feedback regulator in ox-LDL-stimulated THP-1 macrophage inflammatory responses and lipid uptake, which could affect the development of AS. Zhu et al. [24] suggested that miR155 attenuates apoptosis of ox-LDL-mediated RAW264.7 macrophages by targeting FAS-associated death domain protein (FADD), thus inhibiting the formation of AS plaques. 


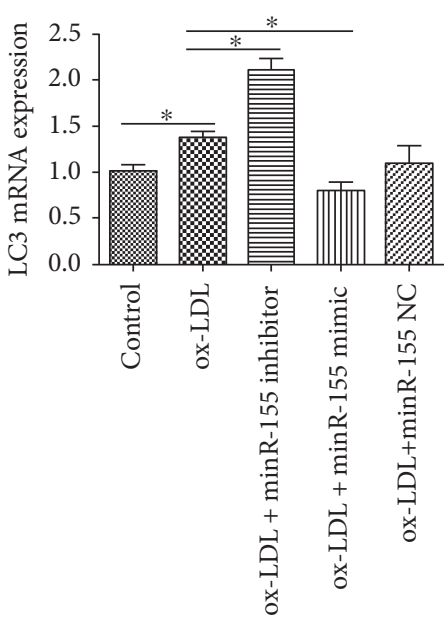

(a)
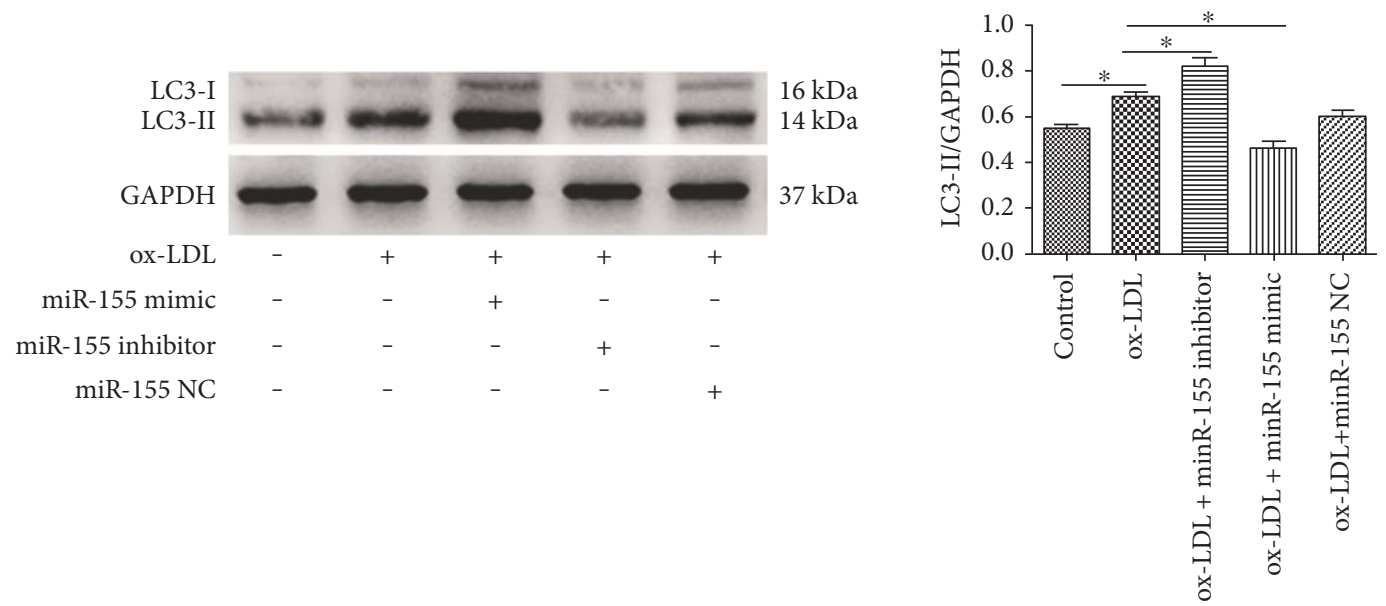

(b)

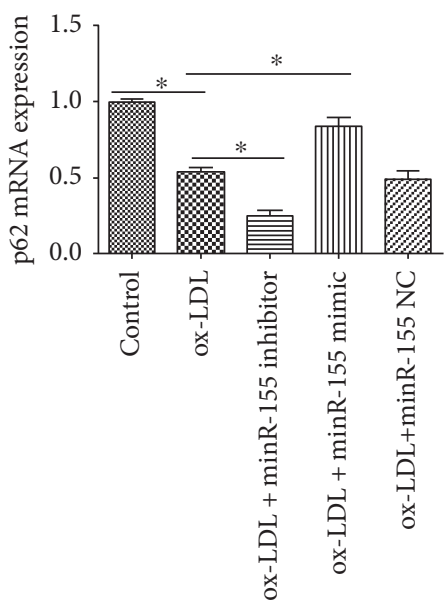

(c)

FIgure 5: Continued. 

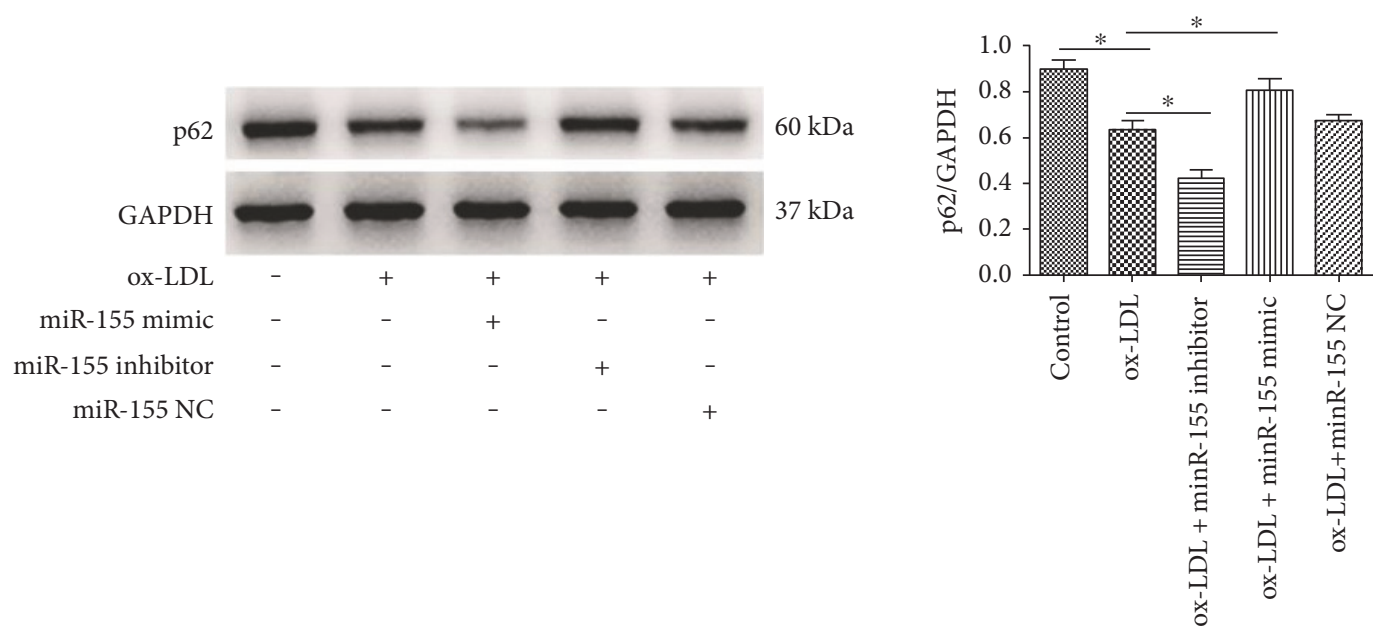

(d)

FIGURE 5: miR-155 regulates LC3 and p62 expression. HUVECs were transfected with miR-155 mimic, miR-155 inhibitor, and mir-155NC and exposed to ox-LDL $(100 \mu \mathrm{g} / \mathrm{ml})$ for $6 \mathrm{~h}$. Cells were harvested for qRT-PCR to detect the expression of mRNA and for Western blot to quantify the protein expression level. (a, c) qRT-PCR analysis of LC3 and p62. Results are the means \pm SD of three separate experiments, ${ }^{*} p<0.05$. (b, d) Western blot analysis of LC3 and p62. Left panels present representative blots of LC3-II and p62; right bar graphs showed statistics of optical density measurements. Results are the means \pm SD of three separate experiments, ${ }^{*} p<0.05$.

Endothelial cells, as the biological basis of angiogenesis, play a crucial role in the pathogenesis of AS [25]. A growing body of evidence has shown that promoting proper autophagy in endothelial cells may be an effective way to improve AS [26, 27]. Our study found that miR-155 has a promoting effect on ox-LDL-induced autophagy in HUVECs. So, we speculated that miR-155 might have an effect on the process of AS by regulating autophagy in endothelial cells.

In conclusion, the present study indicated that ox-LDL induced autophagy and upregulated miR-155 expression in HUVECs. miR-155 has a promoting effect on ox-LDLinduced autophagy. These findings may be able to provide new ideas for studying the possible role of miR-155 on regulating the process of AS. However, further studies, including in vivo experiments, are needed to confirm this role and the mechanism.

\section{Disclosure}

Zhaozhi Zhang and Xudong Pan should be regarded as co-first authors.

\section{Conflicts of Interest}

The authors declare that they have no competing interests.

\section{Authors' Contributions}

Zhaozhi Zhang and Xudong Pan contributed to the work equally.

\section{Acknowledgments}

This study was supported by the National Natural Science Foundation of China (no. 81571112 and no. 81641046) and the Shandong Province Natural Science Foundation (2015GSF118172).

\section{References}

[1] N. Mizushima and M. Komatsu, "Autophagy: renovation of cells and tissues," Cell, vol. 147, no. 4, pp. 728-741, 2011.

[2] M. V. Jain, A. M. Paczulla, T. Klonisch et al., "Interconnections between apoptotic, autophagic and necrotic pathways: implications for cancer therapy development," Journal of Cellular and Molecular Medicine, vol. 17, no. 1, pp. 12-29, 2013.

[3] N. Mizushima, B. Levine, A. M. Cuervo, and D. J. Klionsky, "Autophagy fights disease through cellular self-digestion," Nature, vol. 451, no. 7182, pp. 1069-1075, 2008.

[4] B. Razani, C. Feng, T. Coleman et al., "Autophagy links inflammasomes to atherosclerotic progression," Cell Metabolism, vol. 15, no. 4, pp. 534-544, 2012.

[5] K. Zhao, X. S. Xu, X. Meng, Y. L. Li, J. F. Li, and W. Q. Chen, "Autophagy of monocytes attenuates the vulnerability of coronary atherosclerotic plaques," Coronary Artery Disease, vol. 24, no. 8, pp. 651-656, 2013.

[6] B. Z. Shao, B. Z. Han, Y. X. Zeng, D. F. Su, and C. Liu, "The roles of macrophage autophagy in atherosclerosis," Acta Pharmacologica Sinica, vol. 37, no. 2, pp. 150-156, 2016.

[7] H. I. Im and P. J. Kenny, "MicroRNAs in neuronal function and dysfunction," Trends in Neurosciences, vol. 35, no. 5, pp. 325-334, 2012.

[8] Y. Tang, Y. C. Zhang, Y. Chen, Y. Xiang, C. X. Shen, and Y. G. $\mathrm{Li}$, "The role of miR-19b in the inhibition of endothelial cell apoptosis and its relationship with coronary artery disease," Scientific Reports, vol. 5, p. 15132, 2015. 
[9] D. Santovito, V. Egea, and C. Weber, Small but Smart: MicroRNAs Orchestrate Atherosclerosis Development and Progression, vol. 1861, no. 12, Part B, pp. 2075-2086, 2016, Biochim Biophys Acta.

[10] J. Novak, V. Olejnickova, N. Tkacova, and G. Santulli, "Mechanistic role of MicroRNAs in coupling lipid metabolism and atherosclerosis," Advances in Experimental Medicine and Biology, vol. 887, pp. 79-100, 2015.

[11] M. W. Feinberg and K. J. Moore, "MicroRNA regulation of atherosclerosis," Circulation Research, vol. 118, no. 4, pp. 703-720, 2016.

[12] X. Zhang, H. Shi, S. Lin, M. Ba, and S. Cui, "MicroRNA-216a enhances the radiosensitivity of pancreatic cancer cells by inhibiting beclin-1-mediated autophagy," Oncology Reports, vol. 34, no. 3, pp. 1557-1564, 2015.

[13] L. Zhou, L. Guo, J. Tang, A. Zhang, X. Liu, and G. Xu, "miR-144 regulates BCG- and rapamycin-induced autophagy by targeting Atg4a in RAW264.7 cells," Xi Bao Yu fen Zi Mian Yi Xue Za Zhi, vol. 31, no. 2, pp. 163-167, 2015.

[14] J. Zhu, T. Chen, L. Yang et al., "Regulation of microRNA-155 in atherosclerotic inflammatory responses by targeting MAP3K10," PloS One, vol. 7, no. 11, article e46551, 2012.

[15] X. Li, D. Kong, H. Chen et al., "miR-155 acts as an antiinflammatory factor in atherosclerosis-associated foam cell formation by repressing calcium-regulated heat stable protein 1," Scientific Reports, vol. 6, p. 21789, 2016.

[16] L. Chen, K. Jiang, H. Jiang, and P. Wei, "miR-155 mediates drug resistance in osteosarcoma cells via inducing autophagy," Experimental and Therapeutic Medicine, vol. 8, no. 2, pp. 527-532, 2014

[17] J. Wang, K. Yang, L. Zhou et al., "MicroRNA-155 promotes autophagy to eliminate intracellular mycobacteria by targeting Rheb," PLoS Pathogens, vol. 9, no. 10, article e1003697, 2013.

[18] Y. Chen and D. J. Klionsky, "The regulation of autophagy unanswered questions," Journal of Cell Science, vol. 124, Part 2, pp. 161-170, 2011.

[19] G. Wan, W. Xie, Z. Liu et al., "Hypoxia-induced MIR155 is a potent autophagy inducer by targeting multiple players in the MTOR pathway," Autophagy, vol. 10, no. 1, pp. 70-79, 2014.

[20] M. Nowicki, O. Zabirnyk, N. Duerrschmidt, J. Borlak, and K. Spanel-Borowski, "No upregulation of lectin-like oxidized low-density lipoprotein receptor-1 in serum-deprived EA.hy926 endothelial cells under oxLDL exposure, but increase in autophagy," European Journal of Cell Biology, vol. 86, no. 10, pp. 605-616, 2007.

[22] K. Wu, C. Zhu, Y. Yao, X. Wang, J. Song, and J. Zhai, "MicroRNA-155-enhanced autophagy in human gastric epithelial cell in response to Helicobacter pylori," Saudi Journal of Gastroenterology, vol. 22, no. 1, pp. 30-36, 2016.

[23] R. S. Huang, G. Q. Hu, B. Lin, Z. Y. Lin, and C. C. Sun, "MicroRNA-155 silencing enhances inflammatory response and lipid uptake in oxidized low-density lipoprotein-stimulated human THP-1 macrophages," Journal of Investigative Medicine, vol. 58, no. 8, pp. 961-967, 2010.

[24] G. F. Zhu, L. X. Yang, R. W. Guo et al., "miR-155 inhibits oxidized low-density lipoprotein-induced apoptosis of RAW264.7 cells," Molecular and Cellular Biochemistry, vol. 382, no. 1-2, pp. 253-261, 2013.

[25] R. Ross, "Atherosclerosis-an inflammatory disease," The New England Journal of Medicine, vol. 340, no. 2, pp. 115-126, 1999.
[26] Y. Xiong, G. Yepuri, M. Forbiteh et al., "ARG2 impairs endothelial autophagy through regulation of MTOR and PRKAA/AMPK signaling in advanced atherosclerosis," Autophagy, vol. 10, no. 12, pp. 2223-2238, 2014.

[27] N. Peng, N. Meng, S. Wang et al., "An activator of mTOR inhibits oxLDL-induced autophagy and apoptosis in vascular endothelial cells and restricts atherosclerosis in apolipoprotein E(-)/(-) mice," Scientific Reports, vol. 4, p. 5519, 2014. 


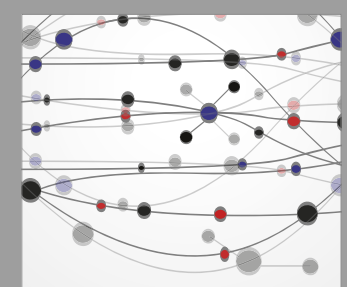

The Scientific World Journal
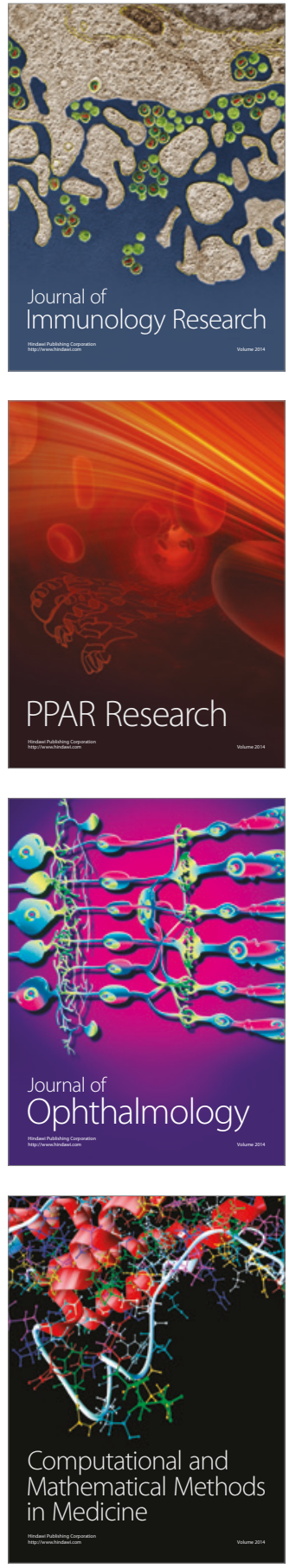

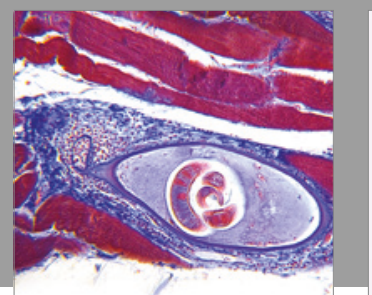

Gastroenterology Research and Practice
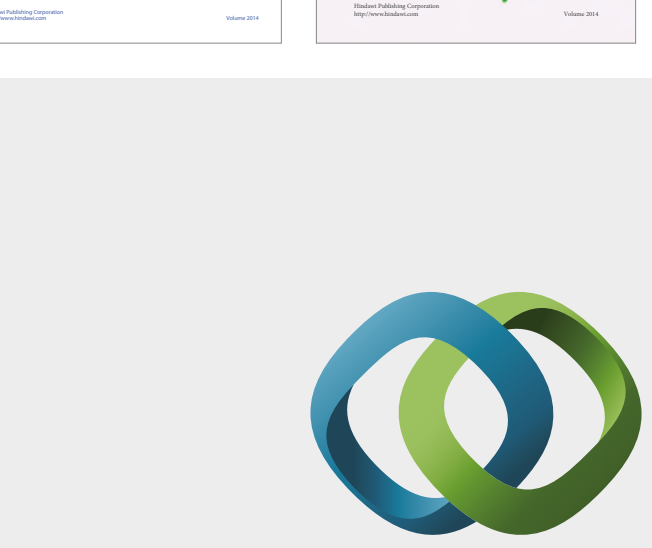

\section{Hindawi}

Submit your manuscripts at

https://www.hindawi.com
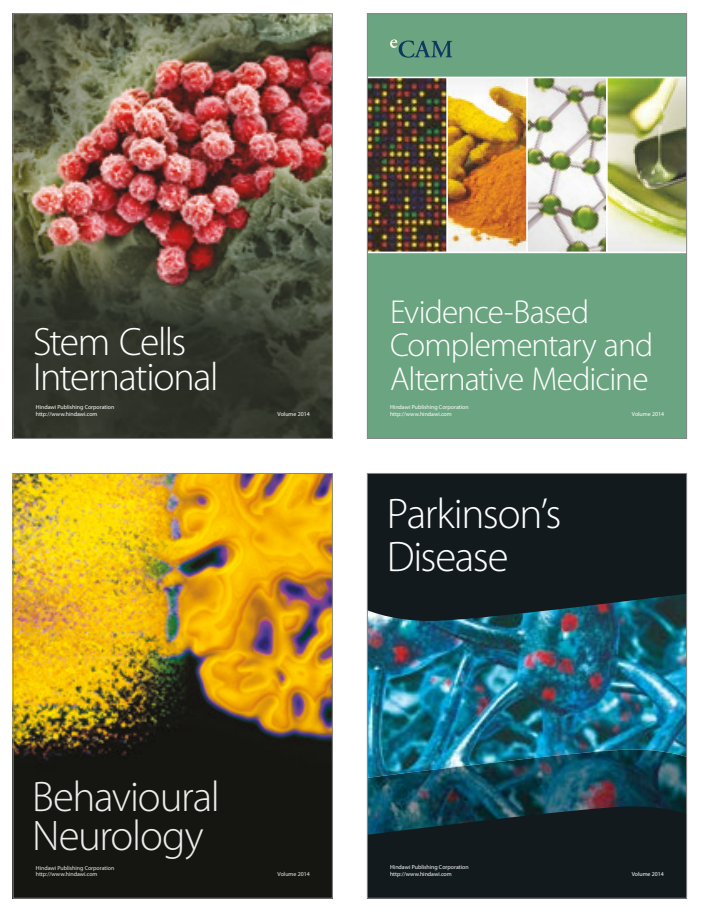
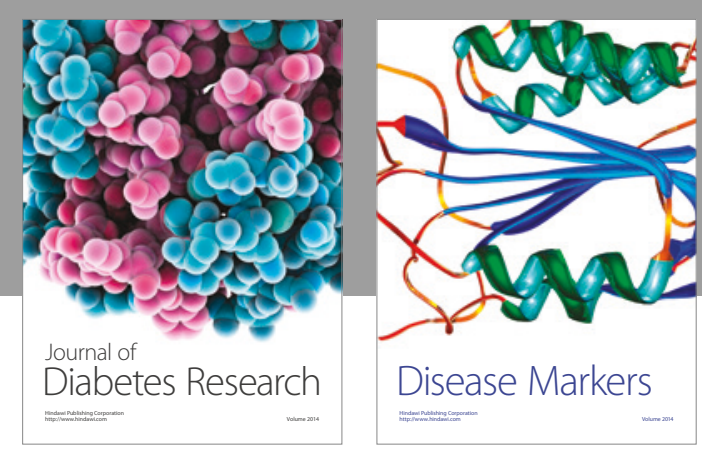

Disease Markers
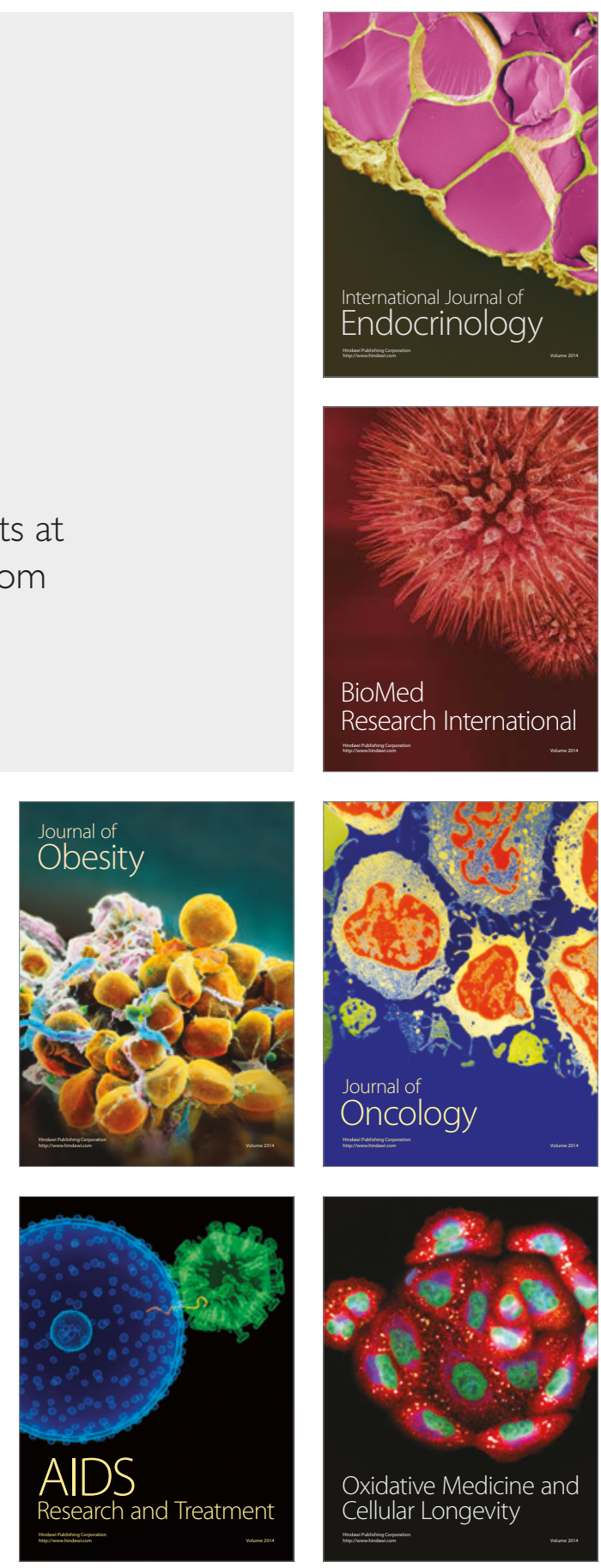